\title{
MENINGIOMAS MÚLTIPLOS EM UM LACTENTE - RELATO DE CASO E REVISÃO DA LITERATURA*
}

\author{
Raimundo Sérgio Furtado de Oliveira', Raimundo Sales Filho²
}

\begin{abstract}
Resumo Neste trabalho é apresentado um caso de meningiomas múltiplos em um lactente de cinco meses de idade, em que o diagnóstico se torna difícil pela apresentação da lesão dominante, de localização selar e supra-selar, com grande área cística na região frontal direita, e que tem grande interesse por sua raridade. Em seguida é apresentada uma revisão da literatura sobre o assunto.

Unitermos: Meningioma. Tomografia computadorizada. Ressonância magnética. Lactente.
\end{abstract}

Abstract Multiple intracranial meningiomas in an infant - case report and review of the literature.

We report a rare case of multiple meningiomas in a 5-month-old infant. Diagnosis was difficult due to the uncommon presentation of the lesions. The main lesion was observed at the sellar and suprasellar regions and a large cystic lesion was also seen in the right frontal region. A review of the literature is also presented. Key words: Meningioma. Computed tomography. Magnetic resonance imaging. Infant.

\section{INTRODUÇÃO}

Meningiomas são, em geral, neoplasias benignas de crescimento lento, derivadas das células meningoteliais (aracnóideas). Crianças podem ser afetadas, mas a maioria dos meningiomas ocorre em adultos ${ }^{(1-4)}$. Nas crianças, além de raros, os meningiomas são diferentes dos tumores do adulto. Por exemplo, áreas císticas são encontradas mais freqüentemente em crianças do que em adultos.

Neste trabalho apresentamos um caso raro de meningiomas múltiplos em um lactente de cinco meses de idade, sua apresentação nos exames de imagem (tomografia computadorizada e ressonância magnética) como massas múltiplas, sendo a maior heterogênea com grande componente cístico, e seus achados histopatológicos e imuno-histoquímicos. Fazemos, também, uma revisão da literatura sobre meningiomas nas crianças.

\section{RELATO DO CASO}

Paciente lactente, de cinco meses de idade, sexo masculino, com história de

\footnotetext{
* Trabalho realizado no Hospital Central do Exército (HCE), Rio de Janeiro, RJ.

1. Médico Radiologista, Chefe do Setor de Ressonância Magnética do HCE.

2. Médico Patologista, Chefe do Laboratório de Anatomia Patológica do HCE.

Endereço para correspondência: Dr. Raimundo Sérgio Furtado de Oliveira. Estrada do Capenha, 155/302, Freguesia, Jacarepaguá. Rio de Janeiro, RJ, 22743-041. E-mail: rasfo@bridge.com.br

Aceito para publicação em 10/4/2001
}

aumento progressivo do tamanho da cabeça, vômitos, nistagmo e "olhar do sol poente". Há 45 dias a mãe vinha notando que a criança apresentava aumento do tamanho da cabeça, com piora nas últimas duas semanas. O paciente se apresentou com nistagmo, "olhar do sol poente", vômitos (três episódios/dia), recusa da alimentação e hipoatividade.

Exame físico: paciente prostrado, hipoativo, hipocorado, hidratado, eupnéico, afebril, acianótico, perfusão periférica satisfatória, fontanela anterior hipertensa. Ausculta cardíaca: ritmo cardíaco regular em dois tempos, bradicárdico, bolhas normofonéticas, sem sopros. Ausculta pulmonar: murmúrio vesicular universalmente audível, sem ruídos adventícios. Abdome: flácido, indolor, sem visceromegalias. Genitália: masculina, compatível com a idade. Membros: sem edemas. Peso atual: 6.470 gramas. Perímetro cefálico: $50 \mathrm{~cm}$.

Nasceu de parto normal, a termo, sem intercorrências. Pré-natal sem alterações. Chorou quando nasceu. Sem história de cianose e icterícia. Alta com 24 horas de vida. Peso ao nascer: 2.190 gramas; estatura de $44 \mathrm{~cm}$. Mãe com 23 anos de idade, GI/PI/A0, saudável. Pai com 26 anos de idade, saudável. Avó materna hipertensa. Alimentação: leite materno.

Foram realizados exames laboratoriais, que mostraram: hemácias: 4,37 milhões $/ \mathrm{mm}^{3}$; hemoglobina: $11 \mathrm{~g} / \mathrm{dl}$; leucócitos totais: $6,8 \mathrm{mil} / \mathrm{mm}^{3}$; glicose: $95 \mathrm{mg}$ / dl; uréia: $24,3 \mathrm{mg} / \mathrm{dl}$; creatinina: $0,3 \mathrm{mg}$ / dl; sódio: $136 \mathrm{mEq} / \mathrm{l}$; potássio: 5,1 mEq/ 1; coagulograma sem alterações; VDRL: não-reagente; anticitomegalovírus: $\mathrm{IgG}$ $=38 \mathrm{UA} / \mathrm{ml}$ e IgM negativo; anticorpos para toxoplasmose: $\mathrm{IgG}=7,7 \mathrm{UI} / \mathrm{ml}$ e $\mathrm{IgM}=0,1 \mathrm{UI} / \mathrm{ml}$; anticorpos anti-herpes simplex: IgG reativo e IgM não-reativo.

A tomografia computadorizada sem contraste (Figura 1) mostrou grande lesão expansiva supra-selar, com extensão à região frontal direita, de densidade mista (áreas isodensas e áreas hipodensas), alargamento dos espaços liquóricos e dilatação ventricular. Não foi realizada a fase contrastada, tendo sido indicada ressonância magnética.

A ressonância magnética (Figuras $2 \mathrm{a}$ 7) mostrou lesão expansiva centrada na região selar e hipotalâmica, medindo 6,1 $\times 5,3 \times 4,8 \mathrm{~cm}$, com sinal heterogêneo, e após a utilização do meio de contraste (gadolínio-DTPA) evidenciou área irregular de intensa impregnação (sólida) e área não-captante extrínseca (cística). Observaram-se, ainda, quatro outras lesões expansivas extra-axiais, que se impregnaram pelo meio de contraste: duas junto à tenda do cerebelo, com ampla base de implantação e extensão dural, uma junto ao recesso lateral direito do quarto ventrículo e uma junto ao contorno posterior da junção bulbo-medular. A ressonância magnética mostrou, ainda, dilatação ventricular, coleção subdural fronto-temporo-parietal direita, com sinal diferente do sinal do líquor e erosão na mastóide direita. 


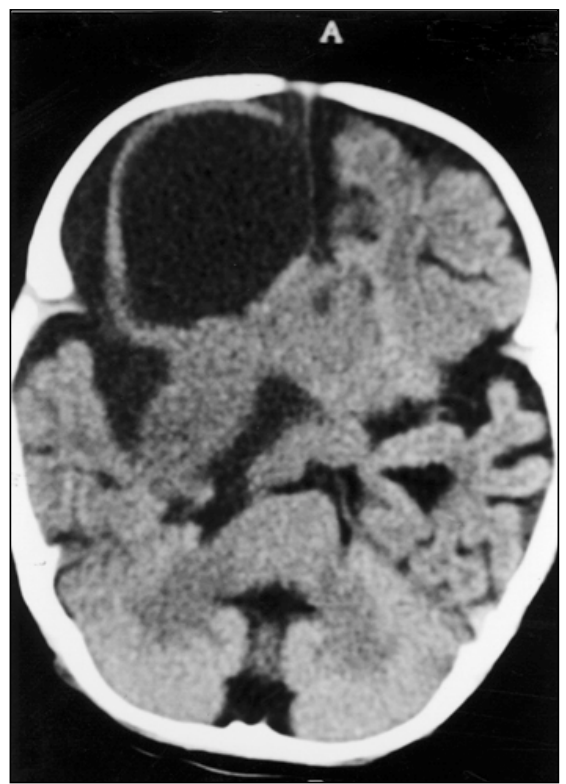

Figura 1. Tomografia computadorizada mostrando lesão expansiva na região supra-selar predominantemente sólida, com extensão hipodensa ao lobo frontal direito (cística).

O paciente foi submetido a cirurgia, tendo sido realizada ressecção parcial da lesão dominante, cujo exame anatomopatológico revelou, na macroscopia, vários fragmentos irregulares de tecido pardacento e elástico, medindo, em conjunto, $5,0 \times 4,0 \times 1,5 \mathrm{~cm}$. Microscopicamente, observou-se neoplasia de linhagem meningotelial formada por feixes entrelaçados de células fusiformes alongadas. $\mathrm{O}$ estudo imuno-histoquímico revelou positividade para antígeno de membrana epitelial e ausência de expressão para proteína S-100.

Diagnóstico: meningioma fibroblástico.

\section{DISCUSSÃO}

O meningioma é um tumor benigno de crescimento lento das meninges e é o tumor primário não-glial mais comum, sendo, basicamente, um tumor do adulto. É o segundo tumor cerebral primário nos adultos, com $15 \%$ a $20 \%$ de freqüência, precedido apenas pelos gliomas. A incidência total na população é de 2,3/ 100.000 habitantes $^{(5)}$, com pico de ocorrência entre 40 e 60 anos de idade, sendo duas a quatro vezes mais freqüente em mulheres. Somente $1 \%$ a $2 \%$ ocorre

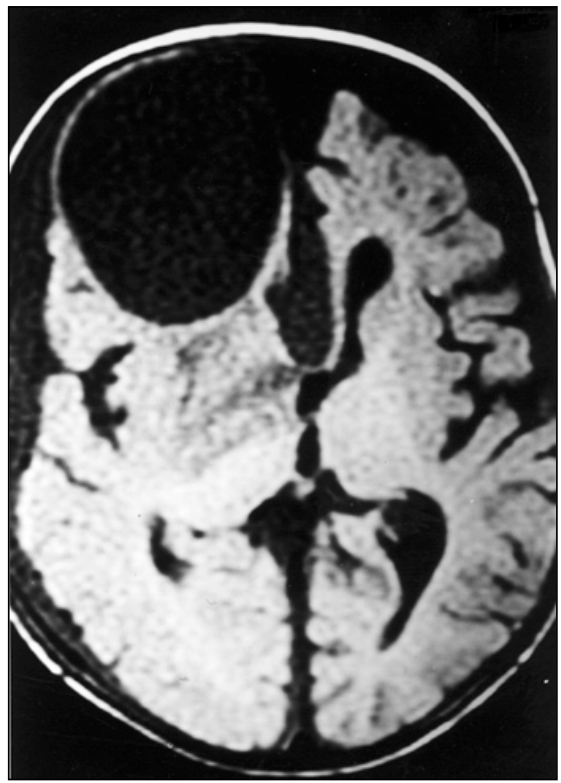

Figura 2. Ressonância magnética, seqüência pesada em T1 no plano axial mostrando, principalmente, a porção cística frontal direita da lesão.

em crianças, na idade de um a 16 anos. Os tumores meníngeos (meningioma e tumores meningeais malignos maldiferenciados) representam $1,6 \%$ de todos os tumores do sistema nervoso central até os 16 anos. É, portanto, o meningioma raro em crianças, e nestas, os meninos são mais afetados que as meninas.

As neoplasias pediátricas do sistema nervoso central incluem tumores gliais e os não-gliais, que diferem significativamente, em localização e biologia, dos tumores dos adultos. A maioria dos tumores cerebrais nas crianças é do tecido neuroepitelial central, ao passo que nos adultos é, em número significativo, das coberturas do sistema nervoso central (meningiomas), dos tecidos adjacentes (adenomas pituitários) e metástases. O tumor maligno mais freqüente do adulto é o glioblastoma multiforme, que tem localização supratentorial, e o mais freqüente da criança é o meduloblastoma, que é infratentorial ${ }^{(6)}$.

As localizações dos meningiomas que são raras nos adultos são comuns nas crianças (por exemplo: fossa posterior) e costumam ter mais alterações císticas que os meningiomas dos adultos.

O prognóstico, nas crianças, é pior do que nos adultos. Nas crianças, os tumo-

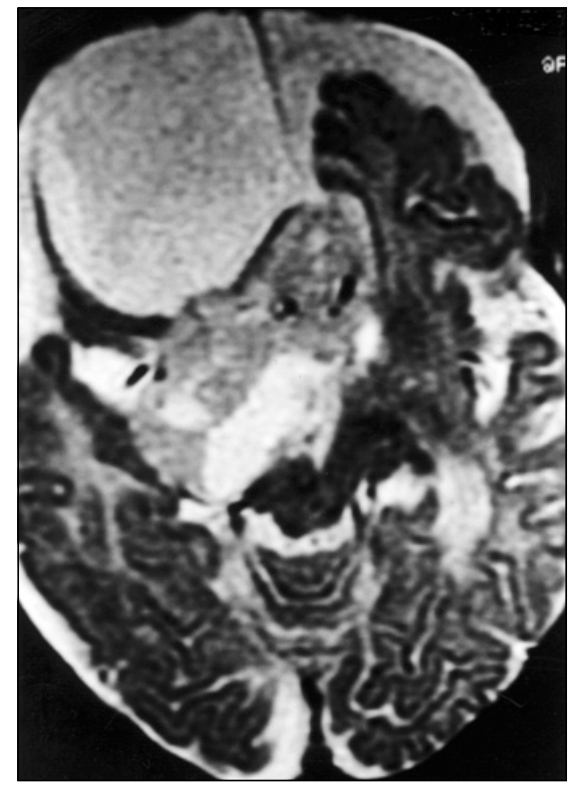

Figura 3. Ressonância magnética, seqüência pesada em T2 mostrando a área de aspecto sólido da massa (sinal intermediário na região supra-selar) e a área cística frontal direita.

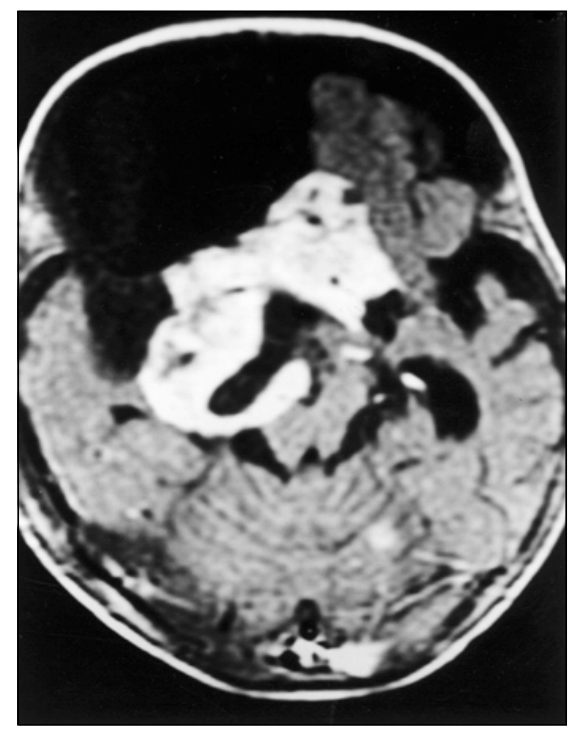

Figura 4. Ressonância magnética, seqüência axial pesada em T1 após uso do meio de contraste à base de gadolínio evidenciando que a porção sólida da lesão se impregna fortemente pelo meio de contraste.

res tendem a crescer mais rapidamente e ter grandes dimensões, sofrem mais freqüentemente alterações malignas e têm maiores taxas de recorrência.

Sheikh et al. ${ }^{(7)}$ descreveram 318 casos de meningiomas. Destes, nove incidiram em crianças e apenas dois eram múltiplos. O meningioma de células claras, 


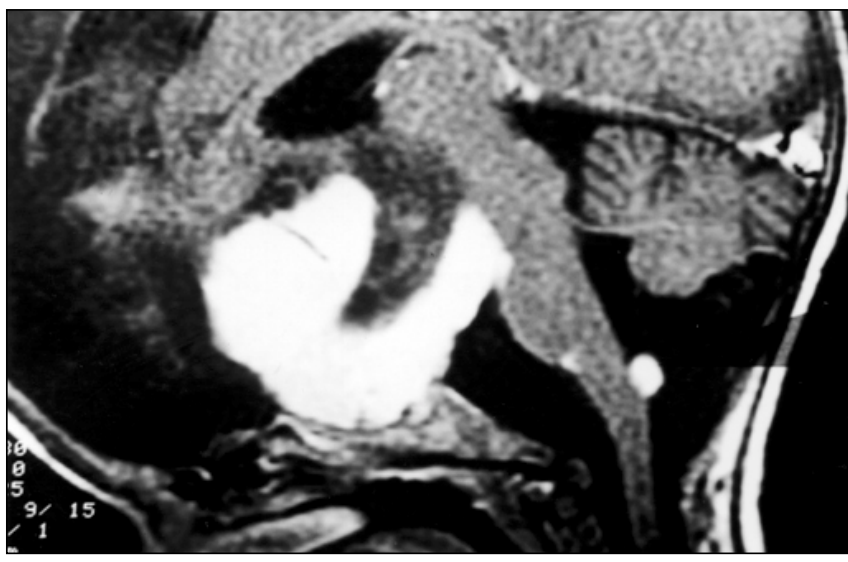

Figura 5. Ressonância magnética, seqüência sagital pesada em T1 após uso do meio de contraste à base de gadolínio mostrando a localização selar e supra-selar da lesão, e também a característica da lesão junto à transição bulbo-pontina.

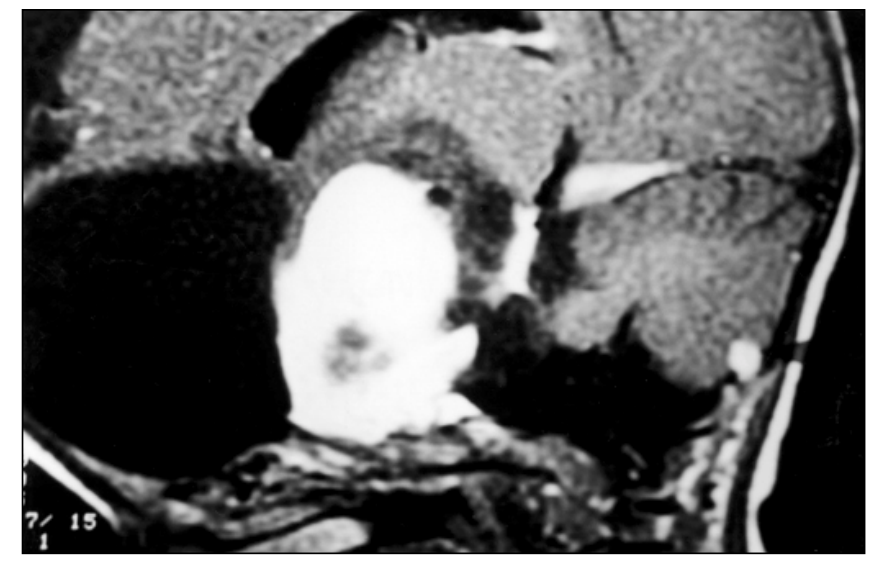

Figura 6. Ressonância magnética, seqüência sagital pesada em T1 após uso do meio de contraste à base de gadolínio, à direita do corte anterior, evidenciando bem a característica cística (hipointensa) e sólida (intensamente impregnada pelo meio de contraste) e sua localização extraaxial. Observam-se também duas outras lesões na fossa posterior, uma com característica extensão dural.

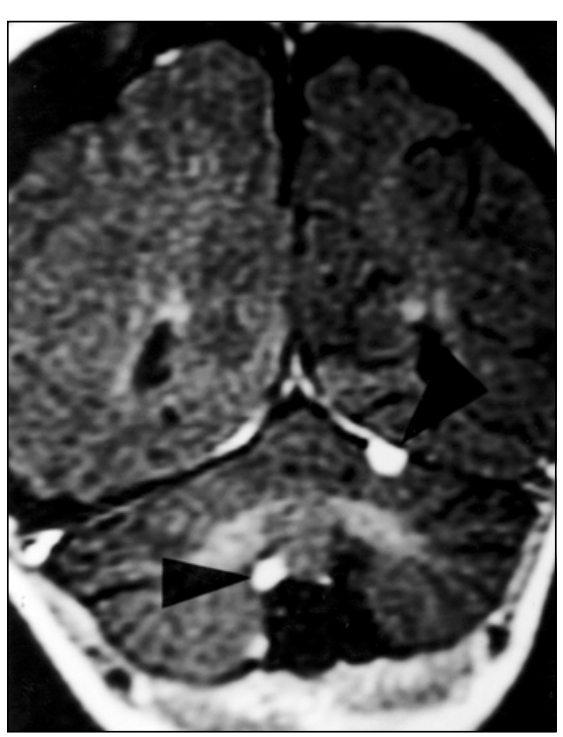

Figura 7. Ressonância magnética, seqüência coronal pesada em T1 após uso do meio de contraste à base de gadolínio mostrando duas lesões junto à tenda do cerebelo, mais bem caracterizada à esquerda, com o típico "sinal da cauda" e a lesão junto ao quarto ventrículo.

tipo mais raro e agressivo, tem predileção pelo grupo etário mais jovem ${ }^{(8)}$.

A ausência de conexão dural é característica de meningiomas pediátricos ${ }^{(9-12)}$. A localização intraventricular $(0,5 \%$ a $1 \%$ dos meningiomas $\left.{ }^{(13)}\right)$ é muito mais comum em crianças ( $15 \%$ a $22 \%$ ) do que em adultos $(0,2 \% \text { a } 4 \%)^{(14,15)}$

Alterações císticas, que tornam o diagnóstico muito mais difícil, são mais comuns no primeiro ano de vida. Mais da

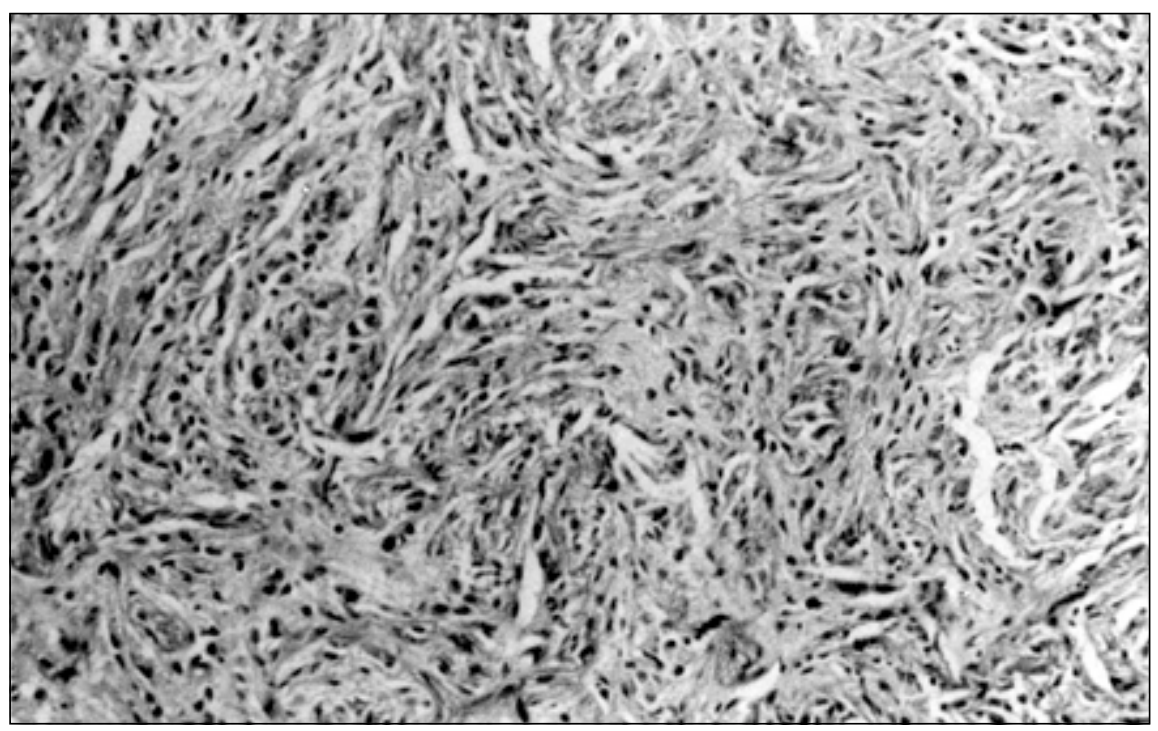

Figura 8. Meningioma fibroblástico com aspecto turbilhonado dos componentes fusocelulares. Hematoxilina-eosina, $160 \times$

metade dos meningiomas císticos em adultos são meningoteliomatosos, enquanto na criança estes são o subtipo menos comum ${ }^{(12,16,17)}$. O meningioma deve, portanto, ser incluído no diagnóstico diferencial de tumores intracranianos císticos em crianças ${ }^{(18)}$.

Existe forte tendência para tumores meníngeos pediátricos terem origem supratentorial e localização intraventricular. A preponderância do sexo feminino em adultos não é vista em crianças ${ }^{(19)}$.

A etiologia dos meningiomas permanece desconhecida, embora esteja, ocasio- nalmente, relacionada com história prévia de irradiação craniana ${ }^{(21-23)}$. Pode-se desenvolver após tratamento radioterápico de crianças portadoras de leuce$\mathrm{mia}^{(24)}$. As crianças parecem ser particularmente sensíveis ao desenvolvimento de meningioma após radioterapia ${ }^{(25)}$. Mais raramente, se relacionam a trauma ${ }^{(26)}$, infecções virais e receptores hormonais, havendo relatos de associação com tumores estrógeno-dependentes, incluindo mama e endométrio ${ }^{(27,28)}$. Influências genéticas, como um locus no cromossomo 22 em paciente com forma central de 
neurofibromatose $(\mathrm{NF} 2)^{(\mathbf{1 0})}$, podem estar relacionadas com a ocorrência de múltiplos meningiomas. Monossomia 22, com ou sem anormalidades cromossômicas, é achado comum no tipo espontâneo.

Nos exames de tomografia computadorizada os meningiomas são tipicamente massas relacionadas a superfície dural, sólidas, lisas ou lobuladas, em sua maioria levemente mais densas que o cérebro e homogêneas, mas algumas podem ser isodensas. Calcificações são encontradas em cerca de $20 \%$ dos casos. Alterações ósseas adjacentes (hiperostose ou destruição óssea) podem ocorrer. Hemorragia, necrose ou alterações císticas são incomuns. Edema peritumoral é visto em mais da metade dos casos, podendo ser extenso. Após a utilização de meio de contraste iodado, observa-se intensa impregnação homogênea em $90 \%$ dos casos. Os $10 \%$ restantes são heterogêneos, com formações císticas.

Na ressonância magnética os meningiomas são massas extra-axiais sólidas homogêneas, em sua maioria iso ou levemente hipointensas em T1 e com sinal variável em T2, e impregnam-se rápida e intensamente pelo meio de contraste paramagnético. O estudo multiplanar permite melhor avaliação da relação do tumor com a meninge e com o cérebro adjacente, mensurando a extensão dural ("sinal da cauda dural"), a reação óssea e os espaços liquóricos (interface) entre o tumor e o cérebro. Nos estudos de espectroscopia in vivo por ressonância magnética os meningiomas típicos geralmente não têm $\mathrm{N}$-acetil-aspartato, podem ou não ter pico de colina (às vezes têm picos extremamente altos, principalmente em recorrência) e possuem pico de alanina como achado mais característico. O lactato pode estar aumentado ${ }^{(\mathbf{2 0 )}}$.

Morfologicamente, os meningiomas são, em sua maioria, massas apresentando larga base de fixação dural. As lesões fibrosas têm maior consistência, enquanto calcificações e ossificações conferem resistência arenosa aos cortes. Áreas amareladas e semigelatinosas correspondem, respectivamente, a lipidização e acúmulo de mucina. Metaplasias condróides, mais raras e focais, não são discerníveis à macroscopia.
Microscopicamente, os meningiomas são tão heterogêneos que desafiam classificações rígidas. A maioria dos espécimes corresponde a um dos subtipos histológicos a seguir descritos, embora poucos ocorram de forma pura.

Os meningiomas podem ser classificados em meningotelial (sincicial), transicional e fibroblástico, além de variantes como microcística, psamomatosa, secretora, linfoplasmocítica, cordóide ${ }^{(\mathbf{2 9 )}}$, metaplásica e papilar. Um único tumor pode ter vários padrões, contudo deverá ser classificado de acordo com o predominante. Às vezes, o termo angiomatoso é aplicado, devendo-se ter o cuidado de se distinguir de angioblástico, que é, em muitas situações, usado para designar hemangiopericitoma meningeal.

Os meningiomas fibroblásticos podem mimetizar schwannomas. Imuno-histoquimicamente, embora possam apresentar reatividade para a proteína S-100, geralmente o fazem de forma fraca e focalmente, ao contrário do padrão difuso de positividade para a proteína S-100 encontrado nos schwannomas. Estes últimos, por outro lado, não apresentam positividade para o antígeno de membrana epitelial, o qual é ótimo marcador para meningioma, contribuindo efetivamente no diagnóstico diferencial entre os dois tumores.

\section{REFERÊNCIAS}

1. Davidson GS, Hope JK. Meningeal tumors of childhood. Cancer 1989;63:1205-10.

2. Deen HG Jr, Scheithauer BW, Ebersold MJ. Clinical and pathological study of meningiomas of the first two decades of life. J Neurosurg 1982; 56:317-22.

3. Ferrante L, Acqui M, Artico M, Mastronardi L, Rocchi G, Fortuna A. Cerebral meningiomas in children. Childs Nerv Syst 1989;5:83-6.

4. Hope JK, Armstrong DA, Babyn PS, et al. Primary meningeal tumors in children: correlation of clinical and CT findings with histologic type and prognosis. AJNR 1992;13:1353-64.

5. Bondy M, Ligon BL. Epidemiology and etiology of intracranial meningiomas: a review. J Neurooncol 1996;29:197-205.

6. Yachnis AT. Neuropathology of pediatric brain tumors. Semin Pediatr Neurol 1997;4:282-91.

7. Sheikh BY, Siqueira E, Dayel F. Meningioma in children: a report of nine cases and review of the literature. Surg Neurol 1996;45:328-35.

8. Shih DF, Wang JS, Pan RG, Tseng HH. Clear cell meningioma: a case report. Chung Hua I Hsueh Tsa Chih (Taipei) 1996;57:452-6.

9. Kohama I, Sohma T, Nunomura K, Igarashi K, Ishikawa A. Intraparenchymal meningioma in an infant - case report. Neurol Med Chir (Tokyo) 1996; 36:598-601.

10. Mandai K, Tamaki N, Kurata H, Eguchi T. The clinical analysis of pediatric meningioma: 5 cases. No Shinkei Geka 1997;25:131-6.

11. Chidambaram B, Balasubramaniam V. Meningioma without dural attachment in a child. Childs Nerv Syst 1997;13:639-41.

12. Teo JG, Goh KY, Rosenblum MK, Muszynski CA, Epstein FJ. Intraparenchymal clear cell meningioma of the brainstem in a 2-year-old child: case report and literature review. Pediatr Neurosurg 1998;28:27-30.

13. Kloc W, Imielinski BL, Wasilewski W, Stempniewicz M, Jende P, Karwacki Z. Meningiomas of the lateral ventricles of the brain in children. Childs Nerv Syst 1998;14:350-3.

14. Pau A, Dorcaratto A, Pisani R. Third ventricular meningiomas of infancy. A case report. Pathologica 1996;88:204-6.

15. Sudha K, Karak AK, Sharma MC, Mathur M, Sarkar C. Assessment of proliferative potential of meningiomas using PCNA LI and AgNOR counts. Indian J Pathol Microbiol 1998;41:323-30.

16. Lui TN, Lee ST. Cystic meningioma in children: a case report. Chung Hua I Hsueh Tsa Chih (Taipei) 1996;58:303-7.

17. el Abbassi-Skalli A, Chikhaoui N, el Hajjam M, Kadiri R. Cystic meningioma: apropos of 6 cases. J Neuroradiol 1998;25:275-80.

18. Starshak RJ. Cystic meningiomas in children: a diagnostic challenge. Pediatr Radiol 1996;26: 7114.

19. Mallucci CL, Parkes SE, Barber P, et al. Paediatric meningeal tumours. Childs Nerv Syst 1996;12: 582-8.

20. Kinoshita Y, Yokota A. Absolute concentrations of metabolites in human brain tumors using in vitro proton magnetic resonance spectroscopy. NMR Biomed 1997;10:2-12.

21. Cantini R, Burchianti M, Fabrini MG, Valleriani AM, Simi U. Postirradiation meningioma. Childs Nerv Syst 1987;3:382-4.

22. Harrison MJ, Wolfe DE, Lau TS, Mitnick RJ, Sachdev VP. Radiation-induced meningiomas: experience at the Mount Sinai Hospital and review of the literature. J Neurosurg 1991;75:564-74.

23. Saleh J, Silberstein HJ, Salner AL, Uphoff DF. Meningioma: the role of a foreign body and irradiation in tumor formation. Neurosurgery 1991; 29:113-8.

24. Salvati M, Cervoni L, Artico M. High-dose radiation-induced meningiomas following acute lymphoblastic leukemia in children. Childs Nerv Syst 1996;12:266-9.

25. Salvati M, Cervoni L, Puzzilli F, Bristot R, Delfini R, Gagliardi FM. High-dose radiation-induced meningiomas. Surg Neurol 1997;47:435-41.

26. Barnett GH, Chou SM, Bay JW. Posttraumatic intracranial meningioma: a case report and review of the literature. Neurosurgery 1986;18:75-8.

27. Jacobs DH, McFarlane MJ, Holmes FF. Female patients with meningioma of the sphenoid ridge and additional primary neoplasms of the breast and genital tract. Cancer 1987;60:3080-2.

28. Knuckey NW, Stoll J Jr, Epstein MH. Intracranial and spinal meningiomas in patients with breast carcinoma: case reports. Neurosurgery 1989;25: 112-7.

29. Kobata H, Kondo A, Iwasaki K, Kusaka H, Ito H, Sawada S. Chordoid meningioma in a child: case report. J Neurosurgery 1998;88:319-23. 